\title{
621 Identification of Rigid Body Inertia Parameters by Means of an Artificial Seventh Vibration Mode
}

\author{
Robert Kloepper, Naoko Ishizuka, Masaaki Okuma \\ Tokyo Institute of Technology, Department of Mechanical Engineering and Science, \\ 2-12-1 Ookayama, Meguro-ku, Tokyo, Japan
}

\begin{abstract}
Modelling the rigid dynamic behaviour of mechanical structures requires knowledge of the structure's mass, centre of gravity location, and moments of inertia. In cases where the geometry or mass distribution is complex, or when no numerical models of these properties exist, the rigid body parameters must be determined experimentally. In this paper, a new experimental method is introduced. The test structure is suspended in soft wires and a mass-spring element is attached successively to at least three different points. For each attachment, the shape and frequency of the seventh vibration mode are determined and used to solve the equations of motion. Our simulation results indicate that the method can achieve high accuracy if the suspension stiffness is low and the natural frequency of the mass-spring element is well chosen.
\end{abstract}

Key Words: Rigid body parameters, experimental identification, artificial seventh mode

\section{Introduction}

The prediction of the rigid dynamic behaviour of mechanical structures is essential for a wide range of engineering problems such as the simulation and optimisation of handling characteristics of vehicles and aircrafts or in the context of system identification for control engineering. The rigid body behaviour is determined by the structure's overall mass, the centre of gravity location, and six moments of inertia. In many practical cases, these parameters must be determined through physical experiments because no precise numerical models of the structure's shape and density distribution are available.

Various experimental methods have been presented in the literature. The mass and the centre of gravity location can be identified in a straightforward way through static weighting, and the moments of inertia can be derived from the natural frequencies of the structure suspended as a rotational pendulum. Multi-filar pendulums have been successful in achieving high accuracy but require the test structure to be centred very precisely with respect to a platform component [1]. This centring process is difficult and must be repeated for each inertia moment. DURISEK ET AL developed a test bed for vehicles that uses a platform with multiple degrees of freedom constrained by springs [2]. The device is rather complex, but it allows identifying six rigid body parameters in a single set-up.

As an alternative to pendulum mechanisms, the moments of inertia can be derived directly from force-acceleration relationships In the most basic procedure, a motor is used to transmit a moment of force to the test structure and the resulting angular acceleration is measured. HAHN AND NIEBERGALl presented a six axes robot that can measure all rigid body parameters without reconfiguration [3]. In either case, the measurement devices are costly, especially if large structures are to be tested.

A third group of methods is based on the measurement of Frequency Response Functions (FRFs). Typically, an impulse hammer, a number of acceleration sensors, and an FFT analyzer are used for this purpose. In the so-called Modal Parameter Method, the test structure is suspended in soft springs and the six rigid mode shapes are derived from low-frequency FRFs. The rigid body parameters are easily obtained from these mode shapes [4]. In practice, however, it is difficult to suspend the structure in such a way that the six modes can be distinguished [5]. Moreover, it is not always possible to excite all six modes at a time.

Another frequency domain method uses the fact that an unrestrained, perfectly rigid body has a constant FRF called the massline [6]. Given a sufficient number of excitations and acceleration measurements, all rigid body parameters can be derived from massline values. Although the FRF of an actual structure deviates from the massline over most of the spectrum due to the influence of suspension and elastic deformations, it usually comes close to the ideal value at some point between the suspension modes and the first elastic mode. A third method, developed by OKUMA ET $\mathrm{AL}$, uses FRFs to identify the mass and stiffness matrices that represent the dynamic behaviour of the test structure [7]. The rigid body parameters are obtained as a by-product of the mass matrix [8].

In this paper, a new method is introduced that unlike existing approaches requires neither costly measurement equipment nor sophisticated identification algorithms. The method is based on the generation of a seventh vibration mode by means of an auxiliary mass-spring element.

\section{Basic concept of the new method}

The test structure is suspended in soft springs and a single degree of freedom vibration element with spring stiffness $k_{A}$ and mass $m_{A}$ is attached to a point $A$ (Figure 1). The values for $k_{A}$ and $m_{A}$ are chosen in such a way that a seventh mode occurs well separated from both the six suspension modes and the first elastic mode (Figure 2). The auxiliary mass is excited and the acceleration response is measured along the axes $\left\{n^{(i)}\right\}$ of $N$ sensors attached to the test structure, and along the axis $\left\{n^{(A)}\right\}$ of one sensor attached to the mass. To the extent that the seventh mode is not influenced by the suspension (first six rigid modes) and to the extent that it is not influenced by elastic deformations of the structure (elastic modes), the rigid body parameters can be derived accurately from the seventh mode shape and the seventh natural frequency. The equations required for this derivation are discussed in the next section. 


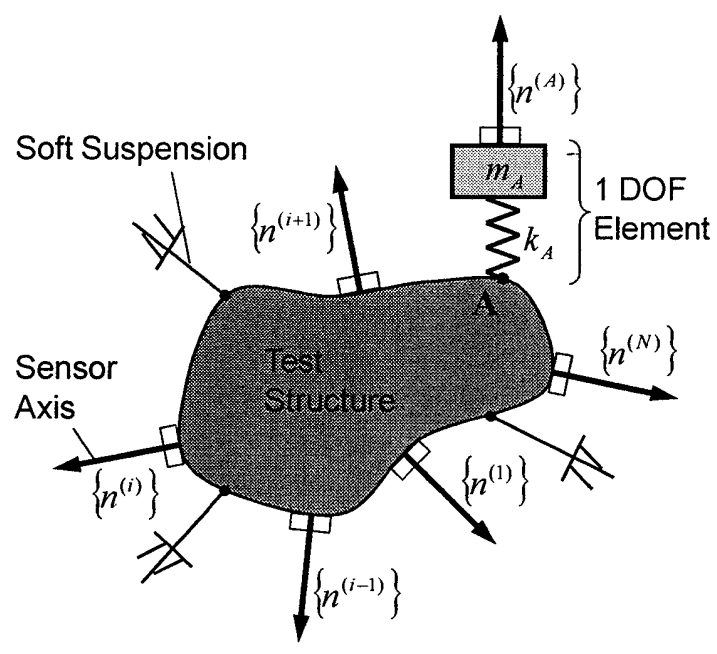

Figure 1: Basic measurement setup

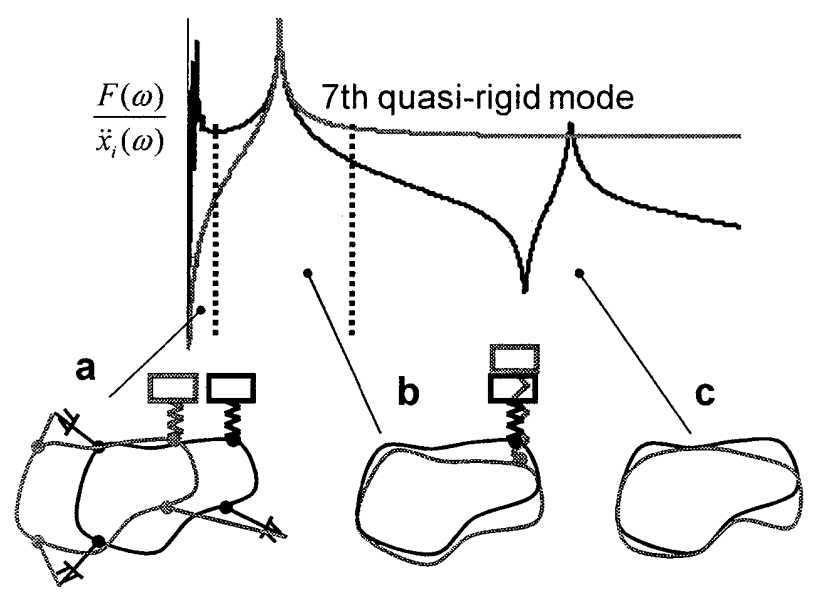

Figure 2: Zones dominated by (a) the suspension modes, (b) the artificial seventh mode, and (c) the elastic modes
Provided that the mass $m_{A}$ of the vibration element is small compared to the test structure inertia, the seventh mode has a predictable natural frequency of

$$
\omega_{7} \approx\left(\frac{k_{A}}{m_{A}}\right)^{1 / 2}
$$

Since the seventh mode can further be expected to be well separated from adjacent modes, the seventh mode shape and the seventh natural frequency may be obtained through simple band-pass filtering of the system's time domain response (cf. Section 5). An FFT analyzer device will no longer be required in this case.

\section{Equations of rigid body identification}

The rigid body parameters to be identified are the mass $m$, the three coordinates $\left\{\zeta^{(G)}\right\}$ of the centre of gravity, and the six independent components of the inertia tensor

$$
[\theta]=\left[\begin{array}{lll}
I_{x x} & & \text { Sym. } \\
I_{y x} & I_{y y} & \\
I_{z x} & I_{y z} & I_{z z}
\end{array}\right] .
$$

\subsection{Equations of motion}

In the first step, the system's motion is described by seven global degrees of freedom $x_{i}(i=1,2, \ldots, 7)$, namely the displacement $x_{A}$ of the auxiliary mass, the translational displacement $\left\{x_{G}\right\}$ of the structure's centre of gravity, and the structure's angular displacement $\{\varphi\}$. With respect to these degrees of freedom, and under the assumption of free rigid behaviour, the system's kinetic energy is given by

$$
E_{k}=\frac{m}{2}\left\{\dot{x}_{G}\right\}^{T}\left\{\dot{x}_{G}\right\}+\frac{1}{2}\{\dot{\varphi}\}^{T}[\theta]\{\dot{\varphi}\}+\frac{m_{A}}{2} \dot{x}_{A}^{2},
$$

and the potential energy stored in the spring is given by
$E_{p}=\frac{k_{A}}{2}\left(\left\{n^{(A)}\right\}^{T}\left(\left\{x_{G}\right\}+\{\varphi\} \wedge\left(\left\{\zeta^{(A)}\right\}-\left\{\zeta^{(G)}\right\}\right)\right)-x_{A}\right)^{2}$.

Using the Lagrange principle

$$
\frac{d}{d t}\left(\frac{\partial E_{k}}{\partial \dot{x}_{i}}\right)-\frac{\partial E_{p}}{\partial x_{i}}=0, \quad i=1,2, \ldots, 7
$$

one equation of motion for each degree of freedom can be obtained from (3) and (4). The next step is to replace $\left\{x_{G}\right\}$ by a kinematic relationship between the displacement $\left\{x_{R}\right\}$ of a reference point and the still unknown coordinates $\left\{\zeta^{(G)}\right\}$ of the centre of gravity. If $R$ is placed in the coordinate origin, the relationship is

$$
\left\{x_{G}\right\}=\left\{x_{R}\right\}+\{\varphi\} \wedge\left\{\zeta^{(G)}\right\}
$$

The equations resulting from the substitution of (6) into (5) can be written in the conventional form of an undamped linear vibration system,

$$
[M]\left\{\begin{array}{c}
\ddot{x}_{A} \\
\left\{\ddot{x}_{R}\right\} \\
\{\ddot{\varphi}\}
\end{array}\right\}+[K]\left\{\begin{array}{c}
x_{A} \\
\left\{x_{R}\right\} \\
\{\varphi\}
\end{array}\right\}=\{0\}
$$

where $[M]$ is the mass matrix and $[K]$ is the stiffness matrix. This system of differential equations has seven solutions, one of which is given by the shape and natural frequency of the seventh mode.

$$
\left(-\omega_{7}^{2}[M]+[K]\right)\left\{\begin{array}{c}
X_{A} \\
\left\{X_{R}\right\} \\
\{\phi\}
\end{array}\right\}_{7}=\{0\}
$$

If the seventh modal parameters are determined experimentally, (8) can be used as a system of six nonlinear equations for the ten unknown rigid body parameters. (The first equation contains only known elements and is therefore redundant.) We found that in order to obtain a unique solution, the mass-spring element must be attached successively to at least three different locations on the test 
structure. This requirement results in a total of $L \geq 18$ equations, each of which ideally satisfies

$$
F_{j}\left(m,\left\{\zeta^{(G)}\right\},[\theta]\right)=0, \quad j=1,2, \ldots, L .
$$

Yet in reality, due to various error sources, the functions $F_{j}$ in the over-determined system (9) cannot all equal zero. Instead, the solution criterion is to minimize the norm of the residuals

$$
\sum_{j=1}^{K} F_{j}^{2}=\min .
$$

We use the Gauss Newton method to solve this nonlinear optimization problem.

\subsection{Kinematic transformation}

In practice, it is almost impossible to measure angular accelerations. Moreover, it is impractical to attach a triaxial sensor directly to the reference point. Consequently, a transformation is needed that derives the reference point mode shapes, $\{\phi\}$ and $\left\{X_{R}\right\}$, from mode shapes determined for a number of translational sensors with arbitrary locations and orientations.

In the same way as in (6), the displacement amplitude $\left\{X_{S}\right\}$ of a sensor located at $\left\{\zeta^{(S)}\right\}$ relates to the displacement of the reference point by

$$
\left\{X_{S}\right\}=\left\{X_{R}\right\}+\{\phi\} \wedge\left\{\zeta^{(S)}\right\} .
$$

If a uniaxial sensor is attached to that point $S$, and if the sensor's orientation is defined by a normal vector $\left\{n^{(i)}\right\}$ that has unity length, the displacement amplitude $D^{(i)}$ actually measured is given by the projection of $\left\{X_{S}\right\}$ onto $\left\{n^{(i)}\right\}$.

$$
D^{(i)}=\left\{n^{(i)}\right\}^{T}\left\{X_{S}\right\}
$$

Substituting (11) into (12) yields one linear equation for the reference point mode shape. For the full set of $N$ sensors, we obtain

$\left\{\begin{array}{c}D^{(1)} \\ \vdots \\ D^{(N)}\end{array}\right\}=\left[\begin{array}{cccc}\left\{n^{(1)}\right\}^{T} & n_{z}^{(1)} \zeta_{y}^{(1)}-n_{y}^{(1)} \zeta_{z}^{(1)} & n_{x}^{(1)} \zeta_{z}^{(1)}-n_{z}^{(1)} \zeta_{x}^{(1)} & n_{y}^{(1)} \zeta_{x}^{(1)}-n_{x}^{(1)} \zeta_{y}^{(1)} \\ \vdots & \vdots & \vdots \\ \left\{n^{(N)}\right\}^{T} & n_{z}^{(N)} \zeta_{y}^{(N)}-n_{y}^{(N)} \zeta_{z}^{(N)} & n_{x}^{(N)} \zeta_{z}^{(N)}-n_{z}^{(N)} \zeta_{x}^{(N)} & n_{y}^{(N)} \zeta_{x}^{(N)}-n_{x}^{(N)} \zeta_{y}^{(N)}\end{array}\right]\left\{\begin{array}{c}\left\{X_{R}\right\} \\ \{\phi\}\end{array}\right\}$

If at least $N=6$ sensors are used, the least squares solution is given by

$$
\left\{\begin{array}{c}
\left\{X_{R}\right\} \\
\{\phi\}
\end{array}\right\}=\left([T]^{T}[T]\right)^{-1}[T]^{T}\left\{\begin{array}{c}
D^{(1)} \\
\vdots \\
D^{(N)}
\end{array}\right\}
$$

\section{Numerical verification}

Since the identification equations assume free rigid behaviour, errors can be expected to increase with the suspension stiffness and with the structure elasticity. The effect depends on the seventh natural frequency (which as stated by Equation 1 can be adjusted through the choice of $k_{A}$ and $m_{A}$.) In order to quantify these

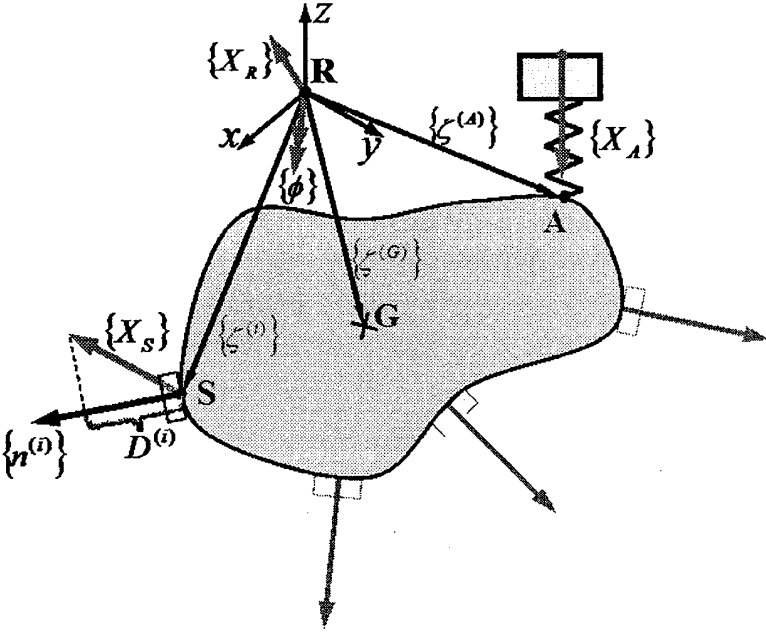

Figure 3: Geometric relationships used in the identification equations

phenomena, the identification method was applied to the finite element model of the beam structure shown in Figure 4.

Initially, the model was assigned with the density and stiffness properties of steel, in which case the first elastic mode occurred at $420 \mathrm{~Hz}$. The structure was suspended by six wires of stiffness $k_{S}$, and a mass-spring element was successively attached to three different points. For each attachment, the seventh mode shape values were calculated for seven coordinates as shown in Figure 4. In a physical experiment, each coordinate would correspond to the axis of an acceleration sensor. The mode shapes and the seventh natural frequency were finally used to solve (10) for the rigid body parameters.

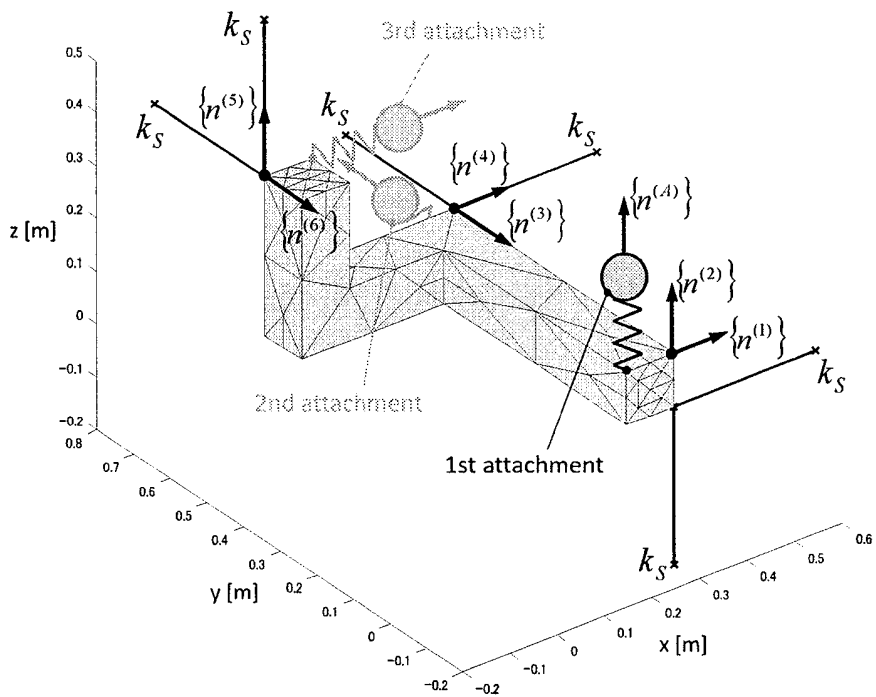

Figure 4: Simulation test case

In order to facilitate the interpretation of the results, the error values $e_{i}$ for the individual rigid body parameters were condensed into a single average value, $\sum_{i=1}^{10}\left|e_{i}\right| / 10$. For the reason that the choice of the coordinate system is arbitrary, the precision of the centre of gravity location was normalized by the structure's longest 
extension in the respective direction. In $x$-direction, for instance, the error value was hence defined by $\Delta \zeta_{x}^{(G)} /\left(0.4 m+\Delta \zeta_{x}^{(G)}\right)$.

Figure 5 shows the results of a series of simulations that investigated the relationship between suspension stiffness, seventh natural frequency, and identification precision. The structure's module of elasticity was held constant at the value for steel. The data confirms that the suspension strongly affects the precision in the low-frequency region. By contrast, the effect for high frequencies is negligible. Nonetheless, with an increasing natural frequency, the precision deteriorates due to the structure's elastic deformation. Over a relatively large band between low and high frequencies, acceptable results are obtained even for $k_{S}=$ $3000 \mathrm{~N} / \mathrm{m}$. For the lowest stiffness value, $k_{S}=200 \mathrm{~N} / \mathrm{m}$, precisions higher than $99.8 \%$ are achieved. The first six modes located between $0.41 \mathrm{~Hz}$ and $1.51 \mathrm{~Hz}$ suggest that this is still a realistic suspension condition.

In a second series of simulations, the influence of the test structure stiffness was examined (Figure 6). For this purpose, the original module of elasticity was scaled by different factors $c$. The suspension stiffness was held constant at a relatively high value of $k_{S}=1000 \mathrm{~N} / \mathrm{m}$. The results show that the impact of the test structure stiffness on the precision increases exponentially with the seventh natural frequency. Yet even for a module of elasticity one fifth that of steel (first elastic mode at $120 \mathrm{~Hz}$ ), more than $99 \%$ accuracy is achieved over a relatively wide frequency range.

In summary, the identification precision was found to be strongly affected by both the structure elasticity in the high-frequency range and by the suspension in the low-frequency range. An optimum balance between both effects can be realized through an appropriate choice of the seventh natural frequency. In that case, the identification results are accurate even for relatively flexible structures. In an experiment, great care should be taken to realize as soft a suspension condition as possible.

Table 1 shows the test structure's actual rigid body parameters and the respective identification errors for one particular configuration $\left(k_{S}=1000 \mathrm{~N} / \mathrm{m}, c=1, m_{A}=1 \mathrm{~kg}\right.$, and $\left.k_{A}=19.410^{3} \mathrm{~N} / \mathrm{m}\right)$. The seventh mode of this system occurred between $22.56 \mathrm{~Hz}$ and $22.78 \mathrm{~Hz}$, depending on the attachment

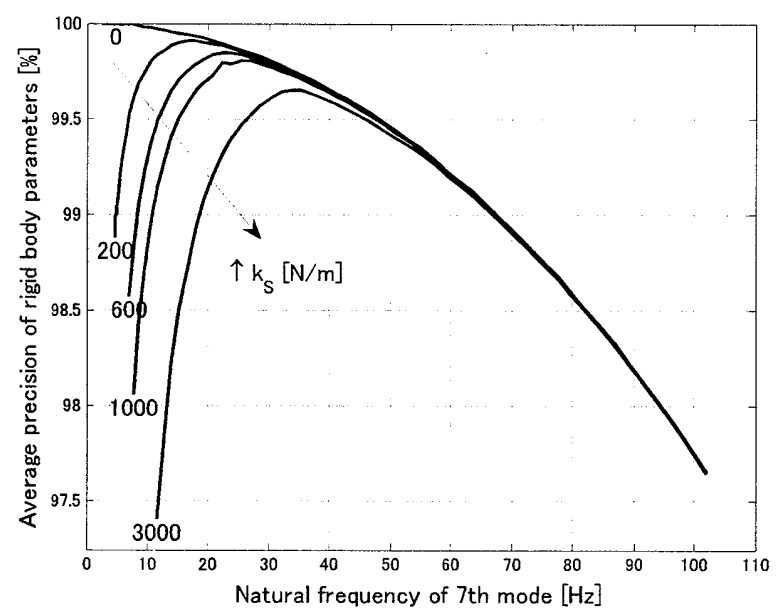

Figure 5: Identification precision as a function of suspension stiffness and seventh natural frequency location. It can be seen that errors for the centre of gravity location are significantly smaller than errors for the inertia parameters. This was found to be a general trend.

\begin{tabular}{|c|r|c|}
\hline & True RBP & Error [\%] \\
\hline$m$ & $86.1300 \mathrm{~kg}$ & 0.0875 \\
\hline$I_{x x}$ & $3.1759 \mathrm{~kg} \mathrm{~s}^{4}$ & 0.4080 \\
\hline$I_{y y}$ & $1.9516 \mathrm{~kg} \mathrm{~s}^{4}$ & 0.2003 \\
\hline$I_{z z}$ & $4.3290 \mathrm{~kg} \mathrm{~s}^{4}$ & 0.1264 \\
\hline$I_{x y}$ & $1.2813 \mathrm{~kg} \mathrm{~s}^{4}$ & 0.1999 \\
\hline$I_{x z}$ & $0.4484 \mathrm{~kg} \mathrm{~s}^{4}$ & 0.7331 \\
\hline$I_{y z}$ & $-0.3203 \mathrm{~kg} \mathrm{~s}^{4}$ & 0.1554 \\
\hline$\zeta_{x}^{(G)}$ & $0.2409 \mathrm{~m}$ & 0.0413 \\
\hline$\zeta_{y}^{(G)}$ & $0.4136 \mathrm{~m}$ & 0.0150 \\
\hline$\zeta_{z}^{(G)}$ & $0.0773 \mathrm{~m}$ & 0.0481 \\
\hline$\frac{1}{10} \sum_{1=1}^{10}\left|\mathrm{e}_{\mathrm{i}}\right|$ & \multicolumn{3}{|c}{$\mathbf{0 . 2 0 1 5}$} \\
\hline
\end{tabular}

Table 1: Correct rigid body parameters and identification errors

\section{Identification of the seventh modal parameters through band-pass filtering}

In Section 2, we suggested the possibility of identifying the seventh modal parameters through band-pass filtering of the test set-up's time domain response. The assumption was based on the predictable natural frequency of the seventh mode and on its wide separation from adjacent modes. Arguably the easiest way of exciting the system is by hitting the auxiliary mass with a hammer. In a numerical model, this type of excitation can be approximated as an initial velocity $v_{0}$ for the auxiliary mass, and zero initial velocity everywhere else. As a test case for the time domain identification, this initial condition was assigned to the same finite element model that led to the results in Table 1 .

The initial velocity was defined as $v_{0}=1 \mathrm{~m} / \mathrm{s}$ and a stiffness proportional damping constant of $\beta=0.01$ was assumed. The time response at each virtual sensor was simulated for a period of $3 \mathrm{~s}$ and then filtered by a band of $10 \mathrm{~Hz}$ to $40 \mathrm{~Hz}$. (Since a digital filter is effective only up to frequencies of half the sampling rate, the time response had to be truncated after the 15 th mode. In a physical

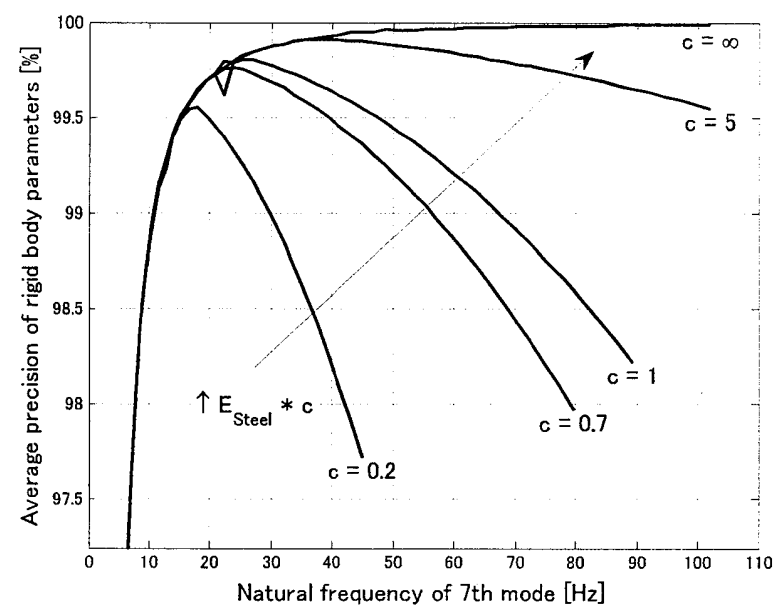

Figure 6: Identification precision as a function test structure stiffness and seventh natural frequency 
experiment, this problem can be avoided by using an analogue filter.)

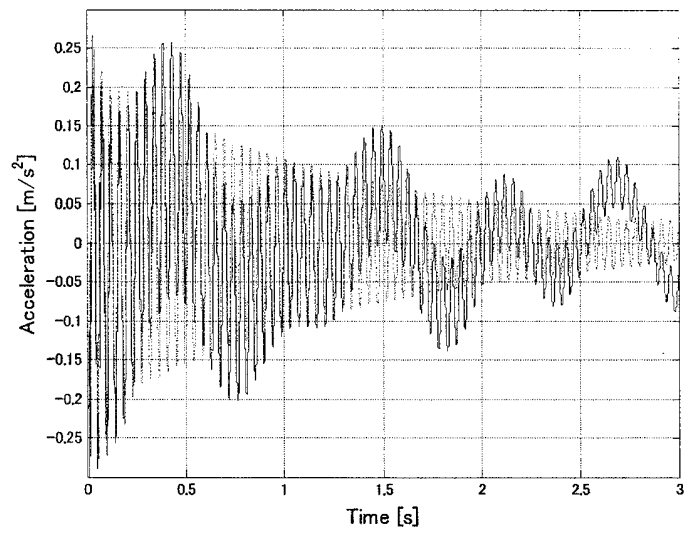

Figure 7: Typical sensor acceleration signal before and after filtering

Ideally, the filtered signal contains only the seventh mode component. In that case, the amplitudes of each half-cycle $k$ are equivalent to the mode shape values $D^{(i)}$ and $X_{A}$ required to solve the identification equations. In order that the values can be averaged over the whole set of $K$ cycles, the amplitudes $a_{k}^{(i)}$ for sensors attached to the structure are normalized by the respective amplitudes $a_{k}^{(A)}$ of the auxiliary mass. Due to the normalization, the values of $X_{A}$ are always one, while those of $D^{(i)}$ are given by

$$
D^{(i)}=\frac{1}{K} \sum_{k=1}^{K} a_{k}^{(i)} / a_{k}^{(A)}, i=1,2, \ldots, N
$$

The errors of the mode shapes identified in this way for the test case are shown in Table 2.

\begin{tabular}{|c|c|c|c|c|c|c|}
\hline & $D^{(1)}$ & $D^{(2)}$ & $D^{(3)}$ & $D^{(4)}$ & $D^{(5)}$ & $D^{(6)}$ \\
\hline$A_{1}$ & $3.2810^{-4}$ & $5.4410^{-4}$ & $7.4810^{-4}$ & $2.8110^{-3}$ & $6.0110^{-3}$ & $1.3410^{-3}$ \\
\hline$A_{2}$ & $1.4910^{-5}$ & $5.3110^{-4}$ & $4.6710^{-5}$ & $1.4910^{-5}$ & $5.3110^{-4}$ & $1.6710^{-4}$ \\
\hline$A_{3}$ & $1.6710^{-4}$ & $7.1410^{-4}$ & $3.1410^{-4}$ & $3.8110^{-5}$ & $2.5910^{-4}$ & $3.1410^{-4}$ \\
\hline
\end{tabular}

Table 2: Errors of the mode shape identification in [\%] for the different sensors and attachment cases

The seventh natural frequency is derived from the average distance between the amplitude points (cf. Figure 8).

$$
\omega_{7}=\frac{1}{\mathrm{~K}-1} \sum_{k=1}^{K-1} \pi / \Delta t_{k}
$$

In the simulation, the frequencies identified for the three respective attachments of the mass-spring element showed errors of $4.0610^{-3} \%, 2.0110^{-3} \%$, and $4.2110^{-4} \%$.

The near-perfect precision of the simulation results suggest that, from a theoretical point of view, band-pass filtering is a viable means of identifying the seventh modal parameters. This finding needs yet to be confirmed by physical experiments.

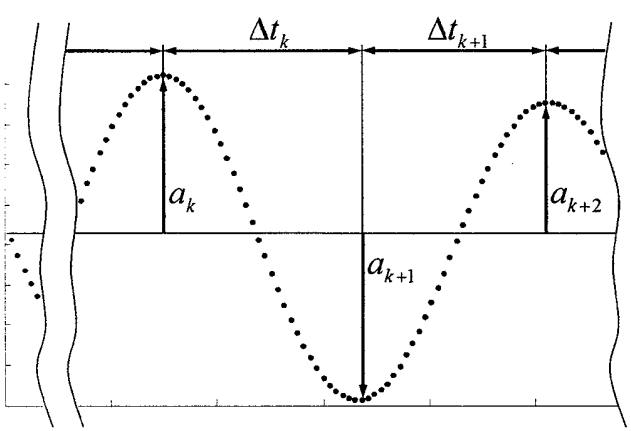

Figure 8: Amplitudes and cycle lengths for modal parameter identification from filtered time domain signal

\section{Conclusion}

This paper introduced a new experimental method for the identification of the rigid body parameters of mechanical structures. The basic principle is the generation of a seventh vibration mode by means of an auxiliary mass-spring element. The method is easy to perform and requires no sophisticated measurement equipment. Our simulation results show that errors due to the simplifying assumption of free rigid behaviour are small if the suspension stiffness is low and if the natural frequency of the mass-spring element is well chosen.

\section{References}

[1] P. L. Ringegni, M. D. Actis and A. J. Patanella (2001). An experimental technique for determining mass inertial properties of irregular shape bodies and mechanical assemblies. Measurement, Volume 29, Issue 1.

[2] Durisek, N.J., Heydinger, G.J., Christos, J.P. and Guenther, D.A. (1997). Land vehicle roll/yaw product of inertia measurement. Transactions of the ASME, Volume 119.

[3] Hahn, H. and Niebergall, M. (2001). Development of a measurement robot for identifying all inertia parameters of a rigid body in a single experiment. IEEE Transactions of Control Systems Technology, Volume 9, Issue 2.

[4] Bretl, J. and Conti, P. (1987). Rigid body mass properties from test data. Proceedings of the 5th International Modal Analysis Conference, IMAC, London, UK.

[5] Almeida, R., Urgueira, A. and Maida, N. (2007). Identification of rigid body properties from vibration measurements. Journal of Sound and Vibration, Volume 299, Issues 4-5.

[6] Fregolent, A. and Sestieri, A. (1996) Identication of rigid body inertia properties from experimental data. Mechanical Systems and Signal Processing, Volume 10, Issue 6.

[7] Okuma, M., Shi, Q. and Oho, T. (1999) Development of the experimental spatial matrix identification method (theory and basic verification with a frame structure). Journal of Sound and Vibration, Volume 219, Issue 1.

[8] Okuma, M., Heylen, W. and Sas, P. (2001). Identification of Rigid Body Properties of 3-D Frame Structure by MCK Identification Method. Proceedings of the 2001 International Seminar on Modal Analysis, Leuven, Belgium. 